\title{
Use of Graphene and Cucurbit[7]uril Electrodes for the Determination of Amantadine in Biological Fluids
}

\author{
C. S. H. Domínguez ${ }^{* \#}$, P. Hernández ${ }^{2}$ \\ ${ }^{1}$ Facultad de Ciencias de la Salud, Universidad Estatal de Milagro, Milagro, Ecuador \\ ${ }^{2}$ Departamento de Química Analítica y Análisis Instrumental, Facultad de Ciencias, Universidad Autónoma de \\ Madrid, Cantoblanco, Madrid, Spain \\ Email: ${ }^{*}$ carmen.hernandez.dominguez@gmail.com
}

Received 27 May 2015; accepted 27 June 2015; published 30 June 2015

Copyright (C) 2015 by authors and Scientific Research Publishing Inc.

This work is licensed under the Creative Commons Attribution International License (CC BY).

http://creativecommons.org/licenses/by/4.0/

cC) (7) Open Access

\begin{abstract}
A differential pulse voltammetry (DPV) method for amantadine (AT) determination is developed. To this end, all the chemical and instrumental variables affecting the determination of amantadine are optimized. These studies have used three types of glassy-carbon electrode, first electrode which has not undergone surface modification or coating, to then modify the working electrode surface with two kinds of suspensions: graphene and graphene-cucurbit[7]uril (CB[7]). From studies of the mechanisms governing the electrochemical response of amantadine, it was concluded that it is an electrochemically system with a diffusive reduction phenomenon. Under optimal conditions and with the appropriate electrode modification, we proceed to study the relation between the peak intensity with the analyte concentration. Thus, we find that when the electrode surface is covered with graphene- $\mathrm{CB}$ [7], two linear sections are obtained, the first one in the concentration range of between $0.05 \mu \mathrm{g} \cdot \mathrm{mL}^{-1}$ and $0.75 \mu \mathrm{g} \cdot \mathrm{mL}^{-1}$; and the second one between $1.00 \mu \mathrm{g} \cdot \mathrm{mL}^{-1}$ and $6.00 \mu \mathrm{g} \cdot \mathrm{mL}^{-1}$, with $\operatorname{Er}(\%)=87$ and R.S.D. $=0.94 \%\left(\mathrm{n}=10\right.$ at $0.5 \mu \mathrm{g} \cdot \mathrm{mL}^{-1}$ level $)$. The minimum detectable amount was $15 \mathrm{ng} \cdot \mathrm{mL}^{-1}$ while a concentration of $44 \mathrm{ng} \cdot \mathrm{mL}^{-1}$ was calculated as determination limit. The optimized method was applied to the determination of amantadine in biological fluids.
\end{abstract}

\section{Keywords}

Glassy-Carbon Electrode, Amantadine, Differential Pulse Voltammetry, Human Urine

\footnotetext{
*Corresponding author.

"http://orcid.org/0000-0002-9754-8050
}

How to cite this paper: Domínguez, C.S.H. and Hernández, P. (2015) Use of Graphene and Cucurbit[7]uril Electrodes for the Determination of Amantadine in Biological Fluids. American Journal of Analytical Chemistry, 6, 623-630. 


\section{Introduction}

Amantadine is a semisynthetic derivative of petroleum hydrocarbons, is formed by rings after six carbon atoms each, it also has an amino group that is electrochemically active [1]-[4] (Figure 1). This amine group is the one that will govern the electrochemical behaviour that we will try to elucidate in this article.

This molecule has been used in the fight against influenza A [5], but no activity against influenza B. The activity of this substance against influenza $\mathrm{A}$ is based on causing interference between the nucleic acids of infectious viral agent and the host cell.

But it was in 1964, when Schwab, England, Poskaner and Young, described the first beneficial effects of amantadine against Parkinson's disease [6].

Despite the many applications that have this molecule, such as influenza A, hepatitis C, Alzheimer and Parkinson, in this article we wanted to focus on its action as influenza agent. To this end, we used biological fluids, namely human urine, as shown in the actual application of the method that is developed here [7].

In this paper, we have decided to take advantage of graphene properties [8]-[10], such as flexibility, self-cooled, high electrical conductivity, elasticity and hardness, and less Joule effect which may be doped by introducing impurities, or diverse substances that modify the graphene conformation (in this case Cucurbit[7]uril), to change its behaviour [11].

Graphene is a carbon allotrope, forming a hexagonal lattice plane, where the carbon atoms are located at the vertices of the lattice, with the type of covalent generated by superimposing the $\mathrm{sp}^{2}$ hybrids bound carbons.

On a microscopic level, it can be considered that, the graphite is formed by superimposing a high number of graphene sheets.

The other substance used as a modifier in this article was the cucurbit[7]uril (CB[7]).

$\mathrm{CB}[7]$ belongs family of cucurbit $[n]$ uril $(\mathrm{CB}[n], \mathrm{n}=5,6,7,8,10)$ consisting of a group of macrocyclic molecules. The name of this group of macrocyclic molecules is due to the similarity of its shape to that of a gourd family Cucurbitaceae [12]-[15].

$\mathrm{CB}[n] \mathrm{s}$ are obtained by the acid-catalyzed condensation of $\mathrm{n}$ glycoluril molecules and formaldehyde to generate molecules with n-glycoluril units bridged by methylene groups. The diameter, and therefore cavity volume cavity depends on the number of glycoluril units repeated.

It is hardly demonstrated that $\mathrm{CB}[n] \mathrm{s}$ are such efficient host molecules in molecular recognition and have a particularly high affinity for charged compounds.

In accordance with mentioned above, in this paper, the electrode of glassy-carbon surface has been modified with, firstly, a suspension of graphene, and secondly, with a suspension of graphene-cucurbit[7]uril to thus develop a method to elucidate the electrochemical behaviour and determine in a simple manner amantadine.

\section{Experimental}

\subsection{Reagents}

Pure standard of amantadine hydrochloride (AT) (>99\%) and cucurbit[7]uril (CB[7]) were purchased from SIGMA-ALDRICH Chemical Co. (St. Louis, USA). graphene was purchased from Nanomaterials. Buffer solutions were prepared with acetic acid, phosphoric and boric acid adjusted to the desired $\mathrm{pH}$ value with sodium hydroxide. Unless otherwise stated, all other reagents were of analytical grade and used as received. Ultrapure water, obtained from Millipore MilliQ System (Waters) was used. All the solutions were prepared just prior to use.

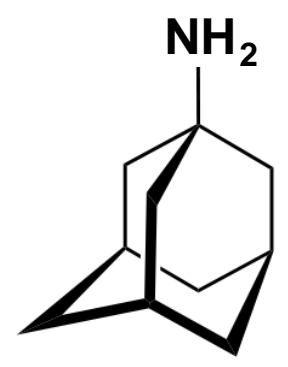

Figure 1. Amantadine structure. 


\subsection{Apparatus}

The electrochemical measurements were performed using a Eco Chemie System General Purpose Electrochemical System (GPES) Potentiostat workstation. A conventional three electrode cell was used. In all experiments, a large area-coiled platinum wire was employed as a counter electrode, an $\mathrm{Ag} / \mathrm{AgCl} / \mathrm{KCl} 3 \mathrm{M}$ as reference electrode and a glassy carbon electrode as the working electrode. For pH adjustment, a pH-meter Methrom C831 was employed.

\subsection{Procedure}

\subsubsection{Solutions Preparation}

AT stock solution $\left(1000 \mu \mathrm{g} \cdot \mathrm{mL}^{-1}\right)$ was prepared in methanol and stored at $4^{\circ} \mathrm{C} \pm 1^{\circ} \mathrm{C}$. A CB[7] stock solution $\left(1.5 \times 10^{-3} \mathrm{M}\right)$ was prepared with $\mathrm{NaCl} 0.2 \mathrm{M}$ and stored at $4{ }^{\circ} \mathrm{C} \pm 1^{\circ} \mathrm{C}$. Both $\mathrm{AT}$ and $\mathrm{CB}$ [7] stock solutions were stored in amber bottles to avoid photodegradation. Prior to analysis, sample bottles were allowed to equilibrate to room temperature.

The preparation of the suspension of graphene was conducted by weighing 0.003 grams of graphene, using as solvent a mixture of 50\% water and dimethylformamide (DMF), reaching a final volume of $1 \mathrm{~mL}$. At Once done, and due to have a suspension as homogeneous as possible, we proceeded to sonicate for 15 - 20 minutes.

To prepare the second suspension, i.e. composed of graphene and $\mathrm{CB}$, we followed the same mechanism as in the case of the suspension of graphene, i.e. graphene $0.03 \mathrm{mg}$ and $0.03 \mathrm{mg}$ of $\mathrm{CB}[8]$, brought to $1 \mathrm{~mL}$ with a 1:1 mixture of water and DMF.

\subsubsection{Sample Preparation}

The application of the method developed in this article was conducted on biological fluids, specifically in human urine.

This sample underwent no treatment of extraction, purification. All what it was done was filtering the sample to remove any particulate matter that may have, as well as to adjust the $\mathrm{pH}$ chosen as the optimum for determination.

\subsubsection{Electrode Surface Preparation}

Once suspensions were prepared with graphene and grapheme-CB[7], we proceeded to modify the surface of the working electrode, through the deposition of 7 - 10 microliters of suspension on the active part of the electrode surface and allowed to dry overnight.

\section{Result and Discussion}

The electrochemical study of the amantadine was performed by differential pulse voltammetry (DPV) in order to determinate the best conditions for its determination.

\section{1. pH Influence}

In order to select the optimum $\mathrm{pH}$ was employed a range of $\mathrm{pH}$ between $\mathrm{pH} 2$ and $\mathrm{pH} 11$. The buffers used were phosphate buffer for $\mathrm{pH}$ 2, 3, 7 and 11; and acetic buffer for $\mathrm{pH} 4$ and 5. The concentration of all of the buffers was $0.5 \mathrm{M}$.

In Figure 2 are represented the voltammograms obtained for the different $\mathrm{pH}$ values. This figure shows that, $\mathrm{pH}=2$ produces a significant signal from an electrochemical standpoint.

The same behavior was observed in the study of the $\mathrm{pH}$ with a glassy-carbon electrode without its active surface has been coated or modified was repeated when the working electrode surface was coated with (1), and graphene (2) with graphene-cucurbit[7]uril.

From the standpoint of electrochemistry and the results, $\mathrm{pH} 2$ was chosen for further studies.

When comparing the recorded voltammograms for the three cases discussed in this article, shows that, although the intensity of the signal, when the electrode is modified with graphene, is much higher, also, the residual current greatly increases, reducing the method selectivity (Figure 3).

Analyzing the behavior of the potential peak, with 1) the unmodified electrode, 2) modified with graphene and 3) modified with graphene and CB[7] can be seen that the potential is shifted to the right, toward more positive potential values. This behavior is indicative of the increased difficulty to oxidize AT. 


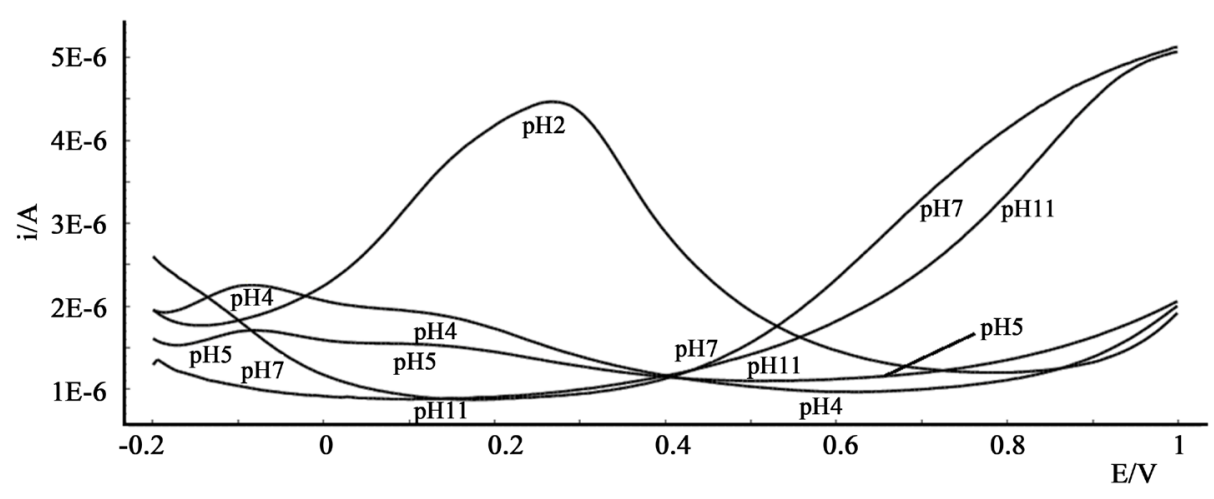

Figure 2. $\mathrm{pH}$ influence with uncoated glassy-carbon electrode. DPV, electrolyte concentration $=0.5 \mathrm{M}$, AT concentration $=10 \mu \mathrm{g} \cdot \mathrm{mL}^{-1}$.

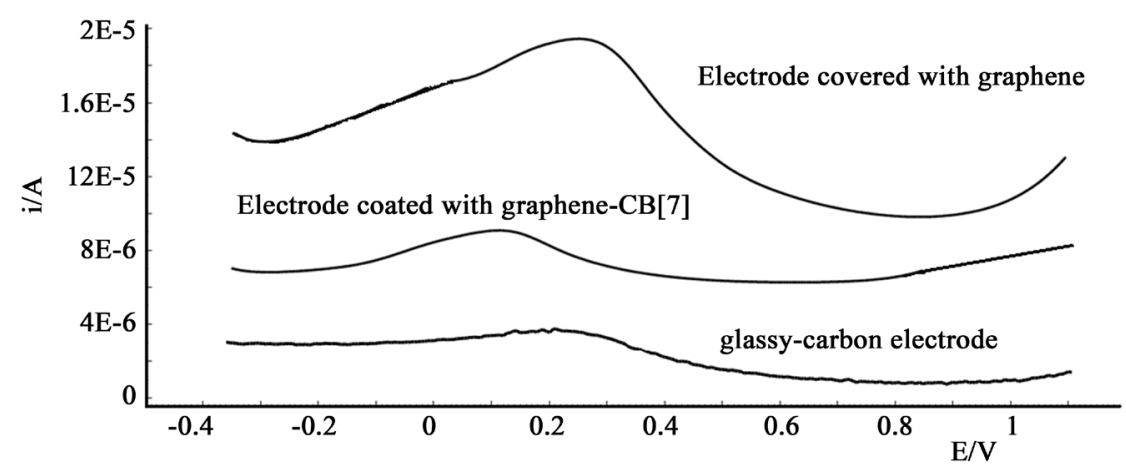

Figure 3. Comparison of electrochemical signals obtained with the three kinds of electrodes used in this paper.

\subsection{Instrumental Variables}

Once the study of the chemical variables, it was proceeded to the optimization of the instrumental variables by differential pulse voltammetry (DPV) and keeping the $\mathrm{pH}$ constant and the concentration of analyte (10 $\left.\mu \mathrm{g} \cdot \mathrm{mL}^{-1}\right)$.

The instrumental variables study was performed with a commercial electrode Glassy-Carbon 1) unmodified, 2) covering the active surface with a suspension graphene and 3) covering the electrode surface with a suspension of graphene and cucurbit[7]uril.

The variables studied were the accumulation time, accumulation potential, scan rate and pulse amplitude, all by DPV.

\subsubsection{Accumulation Potential}

When the influence of accumulation potential in the electrochemical signal was studied, the following results were obtained. In the case of unmodified electrode, the potential of providing the best accumulation signal from the electrochemical standpoint was $-200 \mathrm{mV}$.

For the electrode covered with graphene, the suitable potential accumulation corresponded to a value of -350 $\mathrm{mV}$, but in this case it was observed that as the potential accumulation moved towards more positive values, a preadsorption wave appeared, which provoked the decreased sensitivity of the electrochemical signal of the molecule under study in this paper. For this reason, the influence of the accumulation potential when the surface of the working electrode is covered with a suspension of graphene and $\mathrm{CB}[7]$ was not studied.

The criteria that we followed, and maintained in all studies referenced in this article, to choose the optimal value of the variable under study were: 1) stronger signal; 2) lower semi-peak width and 3) lower residual current.

\subsubsection{Accumulation Time}

A similar event occurred when we studied the influence of accumulation time, the results were 1) unmodified 
electrode $90 \mathrm{~s}$ accumulation time and 2) $120 \mathrm{~s}$ for the electrode covered with graphene, but with increasing accumulation time appeared a preadsorption wave, which lowered the sensitivity of the electrochemical signal.

When the electrode is modified with the suspension composed of graphene-CB[7], and increases the accumulation time, higher than $180 \mathrm{~s}$, there is an electrochemical waveform distortion, and makes it difficult to measure

\subsubsection{Pulse Amplitude}

The procedure followed in this study was the same as in previous studies, i.e. remain constant all variables except the one we are studying at this time, the pulse amplitude.

In addition, we have followed the selection criteria pulse amplitude value discussed above.

According to the discussion in this section, the pulse amplutide values chosen for further study are: 1) for nude electrode surface $50 \mathrm{mV}$; 2) $100 \mathrm{mV}$ for the graphene electrode and 3) $50 \mathrm{mV}$ when the electrode surface is covered with the graphene-CB[7] suspension.

The pulse amplitude follows a linear trend that fit to the equation, 1) uncover electrode $i p(A)=0.134 \Delta E(V)-$ 0.2142 , 2) electrode covered with grapheme $i p(A)=0.2476 \Delta E(V)-0.4848$ and 3) electrode covered with graphene-CB[7] ip $(A)=0.0941 \Delta E(V)-0.1962$, being the range studied $5-200 \mathrm{mV}$

It should be noted that when the electrode surface was covered with graphene and CB[7], we were only allowed to reach a pulse amplitude $50 \mathrm{mV}$ to exceed this value because the peak width and the residual current increased greatly, and this caused a loss of sensitivity in the measures.

\subsubsection{Scan Rate}

When we studied the influence of the scanning velocity was observed that in all three cases studied (electrode unmodified, modified and modified graphene and graphene $\mathrm{CB}[7]$ ), the oxidation process of amantadine was governed by diffusion, because the resulting equation to plot the variation of the peak intensity with respect to the scanning speed was adjusted to the square root, under the range of scan rate between $10 \mathrm{mV} \cdot \mathrm{s}^{-1}$ to $1000 \mathrm{mV} \cdot \mathrm{s}^{-1}$.

(1) unmodified electrode: $i p(\mathrm{~A})=0.1606 \mathrm{v}\left(\mathrm{mV} \cdot \mathrm{s}^{-1}\right)^{(1 / 2)}+1.9787$

(2) electrode covered with graphene: $i p(A)=0.9538 \mathrm{v}\left(\mathrm{mV} \cdot \mathrm{s}^{-1}\right)^{(1 / 2)}+7.8026$

(3) electrode covered with graphene-CB[7]: $i p(\mathrm{~A})=0.6338 \mathrm{v}\left(\mathrm{mV} \cdot \mathrm{s}^{-1}\right)^{(1 / 2)}+2.4019$

The sweep speed values chosen in accordance with the selection criteria outlined above, were: 1 ) for the unmodified electrode $8 \mathrm{mV} \cdot \mathrm{s}^{-1}$, 2) to the electrode $10 \mathrm{mV} \cdot \mathrm{s}^{-1}$ graphene and 3) for the electrode modified with graphene and $\mathrm{CB}[7] 10 \mathrm{mV} \cdot \mathrm{s}^{-1}$.

\subsubsection{Determination of the Stoichiometry of the Complex}

The mole ratio variation method was employed in order to study the stoichiometry of the formed complex between amantadine and cucurbit[7]uril. With this objective, the concentration of amantadine $\left(2.66 \times 10^{-5} \mathrm{M}\right)$ was kept constant and the concentration of $\mathrm{CB}$ [7] was varied in the range of $6.65 \times 10^{-6} \mathrm{M}$ (1 (amantadine):0.25 (CB[7]) and $2.66 \times 10^{-4}(1: 10)$. Solutions were prepared in $0.5 \mathrm{M}$ phosphate buffer $\mathrm{pH}=2$ and the corresponding differential pulse voltammetry voltammograms were recorder under the optimized conditions described above. From the plot Ip, where Ip is the complex AT-CB[7] complex oxidation current vs. the mole ratio of $\mathrm{CB}$ [7], a slope change when the concentration of $\mathrm{CB}$ [7] is $2.66 \times 10^{-5} \mathrm{M}$ indicates that the stoichiometry of the AT-CB[7] complex is 1:1 (Figure 4), which coincides with those obtained by [16].

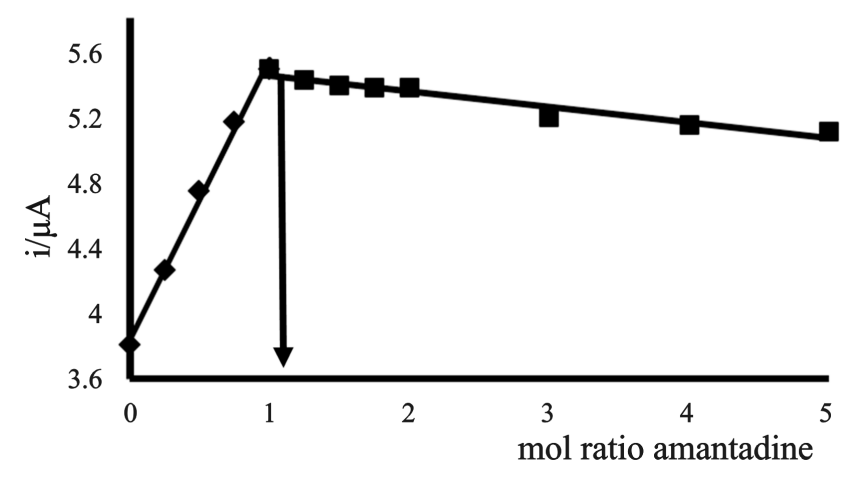

Figure 4. Stoichiometry between amantadine/CB[7]. 
Instrumental variables used in the electrochemical study of complex AT with CB[7] agreed with those obtained in the previous study, that is without cucurbit[7]uril.

\subsubsection{Calibration Curve and Limit of Detection}

After choosing the most suitable measurement conditions for determining the compound under study, it was conducted to study the influence of analyte concentration using the following conditions

(1) Unmiodified electrode: $\mathrm{pH}=2$; accumulation potential $=-200 \mathrm{mV}$; accumulation time $=90 \mathrm{~s}$; scan rate $=$ $8 \mathrm{mV} \cdot \mathrm{s}^{-1}$ and pulse amplitude $=50 \mathrm{mV}$

(2) Electrode modified with graphene: $\mathrm{pH}=2$; accumulation potential $=-350 \mathrm{mV}$; accumulation time $=120 \mathrm{~s}$; scan rate $=10 \mathrm{mV} \cdot \mathrm{s}^{-1}$ and pulse amplitude $=100 \mathrm{mV}$

(3) Electrode coated with graphene-CB[7]: $\mathrm{pH}=2$; scan rate $=10 \mathrm{mV} \cdot \mathrm{s}^{-1}$ and pulse amplitude $=50 \mathrm{mV}$

The analytical performance of the differential pulse voltammetry method developed for amantadine determination was evaluated. From the voltammograms recording for increasing amounts of AT using the optimized parameters described above, it was observed that the peak current increases with the analyte concentration in the range between $0.1 \mu \mathrm{g} \cdot \mathrm{mL}^{-1}$ to $0.6 \mu \mathrm{g} \cdot \mathrm{mL}^{-1}$ with a good linearity according to $i p(A)=0.668 \mathrm{C}\left(\mu \mathrm{g} \cdot \mathrm{mL}^{-1}\right)+2.4458$; $r=0.998$ with an $\operatorname{Er}(\%)=0.75 \%$ and R.S.D. $=1.05 \%\left(\mathrm{n}=10\right.$ at $0.5 \mu \mathrm{g} \cdot \mathrm{mL}^{-1}$ level). The minimum detectable amount was $55 \mathrm{ng} \cdot \mathrm{mL}^{-1}$ while a concentration of $0.1 \mu \mathrm{g} \cdot \mathrm{mL}^{-1}$ was calculated as determination limit.

It was proceeded in the same way when the glassy-carbon electrode surface was coated with graphene and with graphene-cucurbit[7]uril. So, DPV voltammograms were recording for increasing amounts of amantadine in the optimal conditions found for its determination when a glassy-carbon electrode is coated with a suspension of (1) graphene and (2) graphene-CB[7].

Thus, we find that when the electrode surface is covered with graphene, two linear sections are obtained, the first in the concentration range of between $0.05 \mu \mathrm{g} \cdot \mathrm{mL}^{-1}$ and $1 \mu \mathrm{g} \cdot \mathrm{mL}^{-1}$ according to $i p(A)=1.2859 \mathrm{C}\left(\mu \mathrm{g} \cdot \mathrm{mL}^{-1}\right)$ $+3.6206 ; r=0.992$, and the second between $1 \mu \mathrm{g} \cdot \mathrm{mL}^{-1}$ and $10 \mu \mathrm{g} \cdot \mathrm{mL}^{-1}$, according to $i p(A)=0.065 C\left(\mu \mathrm{g} \cdot \mathrm{mL}^{-1}\right)$ $+4.2371 ; r=0.989$. The sensitivity of the method was inferred from the LOD $(3 \sigma)\left(20 \mathrm{ng} \cdot \mathrm{mL}^{-1}\right)$ and LOQ $(3 \sigma)$ (50 $\mathrm{ng} \cdot \mathrm{mL}^{-1}$ ) values. On the other hand, the RSD and Er values of $0.96 \%$ and $0.84 \%$ respectively $(\mathrm{n}=10$ at 5 $\mathrm{ng} \cdot \mathrm{mL}^{-1}$ level) indicated the accuracy and reproducibility of the proposed method.

A similar event occurs when the surface of the working electrode is modified with the suspension graphene and cucurbit[7]uril suspension. That is, when the voltammograms are recorded, two linear sections are obtained, the first one comprised between the values $0.05 \mu \mathrm{g} \cdot \mathrm{mL}^{-1}$ and $0.75 \mu \mathrm{g} \cdot \mathrm{mL}^{-1}$, and a second section between 1.0 $\mu \mathrm{g} \cdot \mathrm{mL}^{-1}$ and $6.0 \mu \mathrm{g} \cdot \mathrm{mL}^{-1}$ (Figure 5). Therefore, in the first range of linearity, an equation is obtained according to $i p(A)=1.1728 C\left(\mu \mathrm{g} \cdot \mathrm{mL}^{-1}\right)+0.6569 ; r=0.991$. And in the second section of linearity, the calibration curve was $i p(A)=0.0417 C\left(\mu \mathrm{g} \cdot \mathrm{mL}^{-1}\right)+1.1723 ; r=0.987$. The determination method has a sensitivity of $\operatorname{Er}(\%)=$ 0.87 and R.S.D. $=0.94 \%\left(\mathrm{n}=10\right.$ at $0.5 \mu \mathrm{g} \cdot \mathrm{mL}^{-1}$ level). The minimum detectable amount was $15 \mathrm{ng} \cdot \mathrm{mL}^{-1}$ while a concentration of $44 \mathrm{ngmL}^{-1}$ was calculated as determination limit.

Finally, for carrying out the method here proposed on a real sample biological, it was imperative picking out which glassy-carbon electrode modification gave the highest selectivity. So, if we compare the voltammograms shown in the figure, it is sighted that although the signal belonging to the electrode covered with graphene-CB[7] is less intense than that provided by the modification with graphene, we have selected the first one owing to the smaller semi-peak width and above all, because the residual current is much lower

\section{Analytical Application}

Because one of the main applications of amantadine, is to treat influenza, in this article we have tried to develop a fast, simple way to determine the degree of removal that has this drug once the patient has ingested it.

Under the selected conditions and with a glassy-carbon work electrode coated with the graphene-cucurbit[7]uril suspension, the proposed method was applied to analyse the amantadine present in human urine doped with a know amount of the studied substance. The human urine sample was just filtered through a cellulose membrane filter with a pore size of $0.45 \mu \mathrm{m}$. This was the only pre-treatment carried out on the sample, which is used in the analytical application of the method here developed.

To perform this experiment it was used the standard addition method, Therefore, sub-samples of $2.0 \mathrm{~mL}$ nonfortified and fortified at different concentration levels $\left(0.5,1.0,2.5\right.$ and $\left.5.0 \mu \mathrm{g} \cdot \mathrm{mL}^{-1}\right)$ were diluted to a final volume of $10.0 \mathrm{~mL}$ with supporting electrolyte and the corresponding square wave voltammograms were recorded. The linear relationship between peak current and AT concentration of AT obeyed the following equation 


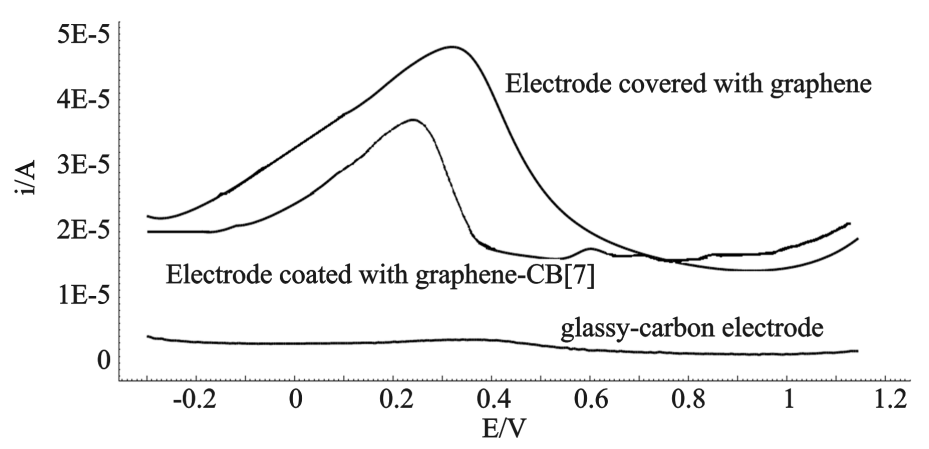

Figure 5. Comparison of the signals obtained with the naked electrode, and with the two modifiers.

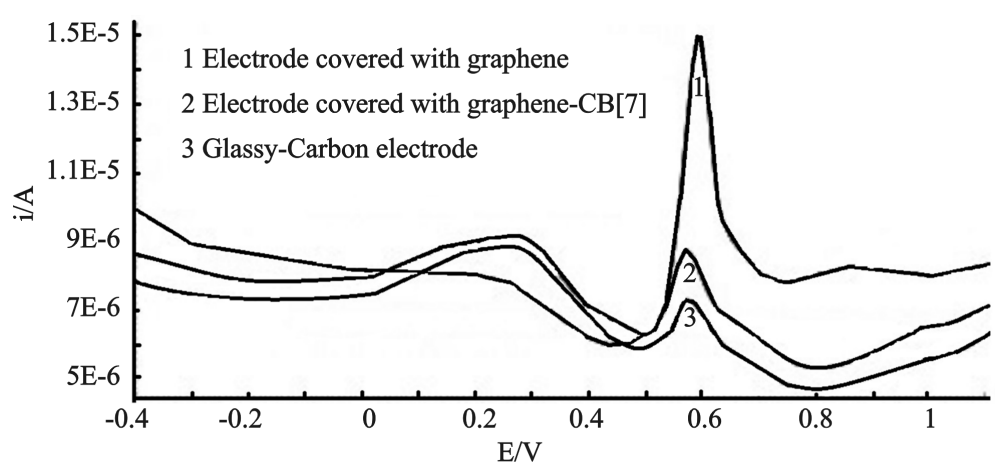

Figure 6. Voltammograms recorded for direct determination of amantadine in human urine. Comparison of the signals obtained with the naked electrode, and with the two modifiers. The concentration of the three solutions was $5 \mu \mathrm{g} \cdot \mathrm{mL}^{-1}$.

ip $=0.3397 C\left(\mu \mathrm{g} \cdot \mathrm{mL}^{-1}\right)+0.6893$, with a correlation coefficient of $R=0.9947$, when the glassy-carbon electrode surface is coated with graphene-CB[7]. It has been chosen this type of coating because, although it does not provide the peak intensities as high as the obtained when the graphene coating is used, the residual current is much smaller and the electrochemical peaks are much better defined. Figure 6 shows the voltammograms obtained.

When the current data obtained are extrapolate into the equation of calibration curve, the concentration of amantadine obtained was $9.57 \mu \mathrm{g} \cdot \mathrm{mL}^{-1}$. Therefore, the degree of extraction of amantadine in human urine is 95.67\%.

The second peak, which appears at $600 \mathrm{mV}$, may be due to many of the compounds present in the urine, because as mentioned before, the sample has not undergone any pretreatment of extraction or derivatization

To corroborate the results obtained electrochemically is employed the method described by A. Sultan, Maha [17], who reacted with ascorbic acid AT in the presence of DMF.

The results obtained by ultraviolet-visible spectroscopy, being the wavelength of $520 \mathrm{~nm}$, are fully in of the line with those obtained by the electrochemical study amantadine. That is, the detected concentration of AT in human urine was $9.87 \mu \mathrm{g} \cdot \mathrm{mL}^{-1}$.

\section{Conclusion}

A sensitive differential pulse voltammetry (DPV) method to analyze amantadine has been developed in this paper. The proposed method can be applied to the determination of the studied molecule, amantadine, in biological fluids excreted by patients who have previously ingested pharmaceutical preparations against influenza, with the inherent advantages of the electrochemical techniques, such as more economical and easy instrumentation with high accuracy and reproducibility as shown from the obtained results

\section{References}

[1] Cui, S.J., Feng, F., Lui, H. and Ma, M. (2007) New Method for High-Performance Liquid Chromatographic Determination of Amantadine and Its Analogues in Rat Plasma. Journal of Pharmaceutical and Biomedical Analysis, 44, 11001105. http://dx.doi.org/10.1016/j.jpba.2007.04.021 
[2] Wang, P., Liang, Y.-Z., Chen, B.-M., Zhou, N., Yu, Y. and Yi, Z.-B. (2007) Quantitative Determination of Amantadine in Human Plasma by Liquid Chromatography-Mass Spectrometry and the Application in a Bioequivalence Study. Journal of Pharmaceutical and Biomedical Analysis, 43, 1519-1525. http://dx.doi.org/10.1016/j.jpba.2006.10.044

[3] Arndt, T., Guessregen, B., Hohl, A. and Reis, J. (2005) Determination of Serum Amantadine by Liquid Chromatography-Tandem Mass Spectrometry. Clinica Chimica Acta, 359, 125-131. http://dx.doi.org/10.1016/j.cccn.2005.03.040

[4] Duh, T.-H., Wu, H.-L., Pan, C.-W. and Kou, H.-S. (2005) Fluorimetric Liquid Chromatographic Analysis of Amantadine in Urine and Pharmaceutical Formulation. Journal of Chromatography A, 1088, 175-181. http://dx.doi.org/10.1016/j.chroma.2005.04.011

[5] Bai, G.-R., Chittaganpitch, M., Kanai, Y., Li, Y.-G., Auwanit, W., Ikuta, K. and Sawanpanyalert, P. (2009) Amantadine- and Oseltamivir-Resistant Variants of Influenza A Viruses in Thailand. Biochemical and Biophysical Research Communications, 390, 897-901. http://dx.doi.org/10.1016/j.bbrc.2009.10.071

[6] Cersosimo, M.G., Scorticati, M.C. and Micheli, F.E. (2000) Amantadine for the Treatment of Levodopa-Induced Dyskinesias in Parkinson's Disease. Medicina (Buenos Aires), 60, 321-325.

[7] Singer, C., Papapatropoulos, S., Uzcategui, G. and Vela, L. (2006) The Use of Amantadine HCL in Clinical Practice: A Study of Old and New Indications. The Journal of Applied Research, 6, 240-245.

[8] Lee, C.G., Wei, X.D., Kyser, J.W. and Hone, J. (2008) Measurement of the Elastic Properties and Intrinsic Strength of Monolayer Graphene. Science, 321, 385-388. http://dx.doi.org/10.1126/science.1157996

[9] Wallace, P.R. (1947) The Band Theory of Graphite. Physical Review, 71, 622. http://dx.doi.org/10.1103/PhysRev.71.622

[10] Guo, Y.J., Wen, D., Zhai, Y.M., Dong, S.J. and Wang, E.K. (2010) Platinum Nanoparticle Ensemble-on-Graphene HYBRID nanosheet: One-Pot, Rapid Synthesis, and Used as New Electrode Material for Electrochemical Sensing. ACS Nano, 4, 3959-68.

[11] Peres, N.M.R. (2010) Colloquium: The Transport Properties of Graphene: An Introduction. Reviews of Modern Physics, 82, 2673-2700. http://dx.doi.org/10.1103/RevModPhys.82.2673

[12] Lagona, J., Mukhopadhyay, P., Chakrabarti, S. and Isaacs, L. (2005) Review: The Cucurbit[n]uril Family. Angewandte Chemie International Edition, 44, 4844. http://dx.doi.org/10.1002/anie.200460675

[13] Kim, K., Selvapalam, N., Ko, Y.H., Park, K.M., Kim, D. and Kim. J. (2007) Functionalized Cucurbiturils and Their Applicvations. Chemical Society Reviews, 36, 267. http://dx.doi.org/10.1039/B603088M

[14] Behrend, R., Meyer, E. and Rusche, F. (1905) Über Condensationproducte aus Glycoluril und Formaldehyd. Justus Liebigs Annalen der Chemie, 339, 1. http://dx.doi.org/10.1002/jlac.19053390102

[15] Liu, S.M., Ruspic, C., Mukhopadhyay, P., Chakrabarti, S., Zavalij, P.Y. and Isaacs, L. (2005) The Cucurbit[n]uril Family: Prime Components for Self-Sorting Systems. Journal of the American Chemical Society, 127, 15959-15967.

[16] Masson, E., Ling, X.X., Joseph, R., Kyeremeh-Mensah, L. and Lu, X.Y. (2012) Cucurbituril Chemistry: A Tale of Supramolecular Success. RSC Advances, 2, 1213-1247. http://dx.doi.org/10.1039/C1RA00768H

[17] Maha, A.S. (2004) Spectrophotometric Determination of Amantadine in Dosage Forms. Current Topics in Analytical Chemistry, 4, 103-109.

\section{Abbreviations}

AT (amantadine)

CB[7] (cucurbit[7]uril)

DMF (dimethylformamide)

AO (ascorbic acid)

DPV (differential pulse voltammetry) 